\title{
Microsecond yellow laser for subfoveal leaks in central serous chorioretinopathy
}

\author{
This article was published in the following Dove Press journal: \\ Clinical Ophthalmology \\ II August 2016 \\ Number of times this article has been viewed
}

\author{
Vikas Ambiya \\ Abhilash Goud \\ Annie Mathai \\ Padmaja Kumari Rani \\ Jay Chhablani
}

Srimati Kanuri Santhamma Retina Vitreous Center, Kallam Anji Reddy Campus, L.V. Prasad Eye Institute, Hyderabad, India
Correspondence: Jay Chhablani L. V. Prasad Eye Institute, L. V. Prasad Marg, Banjara Hills, Hyderabad 500034, India Tel +9l $40306 \mid 2607$ Fax +9| 402354 827| Email jay.chhablani@gmail.com
Purpose: To evaluate the role of navigated yellow microsecond laser in treating subfoveal leaks in nonresolving central serous chorioretinopathy (CSC).

Methods: This prospective study included ten eyes of ten consecutive patients with nonresolving $\mathrm{CSC}$ with subfoveal leaks. All eyes were treated with $577 \mathrm{~nm}$ navigated yellow microsecond laser ( $5 \%$ duty cycle). Key inclusion criteria include a vision loss for a duration of minimum 3 months duration due to focal subfoveal leak on fluorescein angiography. Key exclusion criteria include prior treatment for CSC and any signs of chronic CSC. Comprehensive examination, in addition to low-contrast visual acuity assessment, microperimetry, autofluorescence, spectral domain optical coherence tomography, and fundus fluorescein angiography, was done at baseline, 1, 3, and 6 months after treatment. Rescue laser was performed as per predefined criteria at 3 months.

Results: The average best-corrected visual acuity improved from $73.3 \pm 16.1$ letters to $75.8 \pm 14.0$ $(P=0.69)$ at 3 months and $76.9 \pm 13.0(P=0.59)$ at 6 months, but was not statistically significant. Low-contrast visual acuity assessment (logMAR) improved from $0.41 \pm 0.32$ to $0.35 \pm 0.42$ $(P=0.50)$ at 3 months and $0.28 \pm 0.33(P=0.18)$ at 6 months. Average retinal sensitivity significantly improved from baseline $18.93 \pm 7.19 \mathrm{~dB}$ to $22.49 \pm 6.67 \mathrm{~dB}(P=0.01)$ at 3 months and $21.46 \pm 8.47 \mathrm{~dB}(P=0.04)$ at 6 months. Rescue laser was required only in one eye at 3 months; however, laser was required in three eyes at 6 months.

Conclusion: Microsecond laser is a safe and effective modality for treating cases of nonresolving CSC with subfoveal leaks.

Keywords: CSC, central serous chorioretinopathy, Navilas ${ }^{\mathbb{R}}$, navigated laser, microsecond yellow laser

\section{Introduction}

Central serous chorioretinopathy (CSC), a common chorioretinal disorder, which is characterized by serous retinal detachment in the macula and often associated with serous pigment epithelial detachments. It is known to be self-resolving in many cases. ${ }^{1}$ But refractoriness to treatment and chronicity are known to cause permanent diminution in visual acuity. ${ }^{2-4}$ Such refractory cases are conventionally managed with focal laser. However, conventional laser cannot be applied in subfoveal and juxtafoveal leaks as it carries the risk of central or paracentral scotomas, accidental foveal burn, and choroidal neovascularization. ${ }^{5,6}$ Photodynamic therapy is a viable option in these cases, but it carries its own inherent risk of retinal pigment epithelium (RPE) atrophy, choroidal neovascularization, and choriocapillaris ischemia. ${ }^{7-11}$ Other treatment options include antivascular endothelial growth factors and oral medications.

The micropulse laser differs from conventional thermal laser in that it delivers laser only during 5\%-15\% of duty cycle and therefore prevents collateral thermal damage to the retinal tissue. ${ }^{12,13}$ The mechanism of action of micropulse laser induces 
metabolic effects through the production of mediators with an antiangiogenic and antiedematous action. ${ }^{14,15}$ These properties qualify the micropulse laser to be tried in the treatment of subfoveal and juxtafoveal leaks in CSC.

A more recent type of micropulse laser is the $577 \mathrm{~nm}$ yellow navigated laser (microsecond laser), which could be used to treat subfoveal and juxtafoveal leaks in CSC by titrating the power to a subthreshold level and delivering the laser in micropulse mode. In the present study, we prospectively studied the effect of $577 \mathrm{~nm}$ yellow micropulse navigated laser in treating eyes with persistent CSC with subfoveal leaks.

\section{Methods}

A prospective study was carried out at the L. V. Prasad Eye Institute, Hyderabad, India to evaluate the effect of microsecond laser in treating cases of CSC with subfoveal leaks. Institutional review board approval (L. V. Prasad Eye Institute ethics committee 12-14-120) was obtained for carrying out the study. After detailed explanation of the protocol, all patients signed a formal consent.

The inclusion criteria were: 1 ) age $\geq 18$ years; 2 ) vision loss for a duration of minimum 3 months due to persistent CSC diagnosed by the presence of subretinal fluid (SRF) at the fovea and verified by spectral domain optical coherence tomography (SD-OCT); ${ }^{16} 3$ ) focal subfoveal leak on fundus fluorescein angiography (FFA), defined as a leak within $500 \mu \mathrm{m}$ from the center of fovea.

The exclusion criteria were: 1) history of prior treatment for CSC; 2) absence of any leak or any leak present in addition to the subfoveal leak on FFA; 3) diffuse leak on FFA, RPE atrophy, or any other signs of chronic CSC; 4) vitreoretinal/macular disorders other than CSC currently or in the past; 5) any intraocular procedure in the past 6 months; 6) currently on systemic or topical steroid therapy; 7) any significant media opacity; 8) history of malignant hypertension; 9) pregnancy; 10) currently on any systemic medication such as pioglitazones, which could cause macular edema; 11) evidence of glaucoma; and 12) spherical equivalent $\geq \pm 6 \mathrm{D}$.

A detailed ocular history (onset of symptoms and previous treatment), the demography (age and sex), laterality, and systemic comorbidities (diabetes and hypertension) were recorded. A comprehensive ocular examination was done which included an assessment of the best-corrected distance visual acuity (BCVA) by Early Treatment Diabetic Retinopathy Study (ETDRS) chart, low-contrast visual acuity using COMPlog (24 in widescreen secondary monitor with display resolution of $1,920 \times 1,080$, in a dark room), and
Amsler grid test before dilatation; spherical equivalent of refractive status of the eye; slit lamp biomicroscopy with a noncontact lens; and indirect ophthalmoscopy. Color fundus photographs of the optic disc, macula, and temporal retina $\left(30^{\circ}\right)$ were captured with a mydriatic camera (Zeiss FF450, Carl Zeiss Meditec, Jena, Germany).

\section{FFA}

FFA was performed using fluorescein sodium $20 \%$ and imaging on Navilas ${ }^{\circledR}$ system (OD-OS GmbH, Teltow, Germany) to determine the site of leakage at baseline, and at 3 and 6 months from baseline.

\section{SD-OCT}

The SD-OCT scans were obtained by using Cirrus HD-OCT (Carl Zeiss Meditec, Inc., Dublin, CA, USA) after dilatation of pupil with $0.8 \%$ tropicamide and $5 \%$ phenylephrine eye drops at every visit. The scanning protocol included HD5 line raster, HD single line raster, enhanced depth imaging, and macular cube. Central macular thickness (CMT) was determined automatically and analyzed by OCT software, by generating images using the macular cube $512 \times 128$ scan over $6 \times 6 \mathrm{~mm}$ area, the cube being composed of 128 horizontal examination lines of 512 A-scans each. The CMT was obtained from the $1 \mathrm{~mm}$ central retinal thickness area as described in the ETDRS fields corresponding to the CMT. ${ }^{17}$

The subfoveal choroidal thickness (CT) was measured using the enhanced depth imaging-OCT technique previously described by Spaide et al. ${ }^{18}$ The vertical distance between the hyper-reflective line of Bruch's membrane and the innermost hyper-reflective line of the chorioscleral interface was taken, and the average of the two scans (vertical and horizontal) was regarded as the subfoveal CT. The height of neurosensory detachment (NSD) was measured by the vertical distance between the outermost hyper-reflective margin of the detached neurosensory retina and the hyper-reflective line of the RPE. Scans with a signal strength of $\geq 6$ were used for analysis.

\section{Autofluorescence}

Autofluorescence imaging of the posterior pole (30 degrees; using the Heidelberg retinal angiograph 2 unit, Heidelberg Engineering GmbH, Dossenheim, Germany) was done using the autofluorescence filter.

\section{Microperimetry}

Microperimetry (MAIA ${ }^{\mathrm{TM}}$, Centervue, Padova, Italy) was performed monocularly without mydriasis to assess the retinal sensitivity (RS). The size of the fixation target was selected 
based on the subject's visual acuity. If visual acuity was $<6 / 19.5$ (0.50 logarithm of the minimum angle of resolution $[\log \mathrm{MAR}])$, then the larger fixation target was selected. The 100 grid pattern was selected in the protocol to test the $5^{\circ}$ visual field surrounding the preferred retinal loci, which is determined initially by the machine by showing only fixation target for 10 seconds. The machine tests the retinal thresholds at 37 points across the 50 visual fields using 4-2-1 staircase procedure. Eye with better visual acuity was tested first, followed by the fellow eye. Based on the reliability index, the subjects were asked to repeat the test on the same day whenever necessary. Test was paused whenever the patient felt tired. Microperimetry was done at baseline, and at 1,3, and 6 months from baseline. Using the registration ability of the MAIA ${ }^{\circledR}$, RS was checked at the same points at follow-up visits.

\section{Microsecond laser}

Treatment planning was done using the earliest phase of FFA on Navilas system that showed the leaking spot. Initially, the power of $577 \mathrm{~nm}$ yellow laser was titrated to just produce a mild retinal whitening outside the vascular arcade, using $5 \%$ duty cycle, "test" spot size of $100 \mu \mathrm{m}$ and 0.1 second exposure time. Following this, the navigated microsecond laser was used to place a $5 \times 5$ grid of confluent spots over the area of focal leak, using the same settings with $30 \%$ threshold power.

\section{Rescue laser}

Rescue microsecond laser, with the same settings as in primary laser treatment, was applied at the site of leakage if there was no change or if there was an increase in NSD on SD-OCT at 3 months from baseline.

\section{Outcome measures}

Primary outcome measures included change in BCVA and low-contrast visual acuity at 6 months follow-up compared to baseline. Secondary outcome measures included resolution of NSD, change in RS, and change in CT at the 6-month follow-up compared to baseline.

\section{Statistical analysis}

The BCVA and low-contrast visual BCVA were calculated as ETDRS visual acuity score in the form of number of letters. The changes from baseline in BCVA; height of NSD, CMT, $\mathrm{CT}$; and the average retinal threshold at 1,3, and 6 months were analyzed with Wilcoxon signed-rank test. $P$-value of $<0.05$ was regarded as statistically significant.

\section{Results}

We evaluated ten eyes of ten male patients with treatmentnaïve persistent CSC, with a mean age of $38.2 \pm 4.37$ years. Average duration of symptoms was $7.2 \pm 4.3$ months. All ten eyes showed subfoveal leak on FFA and no other site of leakage was observed. The overall features of the ten eyes at baseline and after follow-up are summarized in Table 1, and those of individual eyes are summarized in Table 2. All eyes were phakic. Figure 1 demonstrates a representative case.

All ten eyes were treated with a sitting of microsecond laser at baseline. The laser power used in our study ranged from 120 to $280 \mathrm{~mW}$. There was an improvement in the BCVA from 73.3 \pm 16.1 ETDRS letters at baseline to $75.8 \pm 14.0$ $(P=0.69)$ letters at 3 months and 76.9 $\pm 13.0(P=0.59)$ letters at 6 months, but the change was not statistically significant at any visit. Similarly, the low-contrast BCVA improved from $79.7 \pm 18.73$ letters at baseline to $86 \pm 16.30$ letters at 6 months, but not statistically significant $(P=0.18)$. However, the average RS on microperimetry was significantly improved from baseline $18.93 \pm 7.19 \mathrm{~dB}$ to $22.49 \pm 6.67 \mathrm{~dB}(P=0.01)$ at 3 months and $21.46 \pm 8.47 \mathrm{~dB}(P=0.04)$ at 6 months.

At 1 month, there was total resolution of SRF in four $(40 \%)$ eyes and reduction in the NSD height in all the remaining eyes.

At 3 months, six eyes (60\%) had no SRF. Of the remaining four eyes, only one eye did not show a progressive

Table I Baseline parameters and changes in follow-up after microsecond laser therapy

\begin{tabular}{lllll}
\hline Parameter & Baseline & I month & 3 months & 6 months \\
\hline Average BCVA (ETDRS letter score \pm SD) & $73.3 \pm 16.1$ & $73.1 \pm 16.3(P=0.97)$ & $75.8 \pm 14.0(P=0.69)$ & $76.9 \pm 13.0(P=0.59)$ \\
Low-contrast BCVA (logMAR) & $0.41 \pm 0.32$ & $0.35 \pm 0.42(P=0.50)$ & $0.45 \pm 0.56(P=0.69)$ & $0.28 \pm 0.33(P=0.18)$ \\
Average retinal sensitivity $(\mathrm{dB}) \pm$ SD & $18.93 \pm 7.19$ & $18.82 \pm 8.46(P=0.16)$ & $22.49 \pm 6.67(P=0.01)^{*}$ & $21.46 \pm 8.47(P=0.04)^{*}$ \\
Average CMT $(\mu \mathrm{m}) \pm$ SD & $298 \pm 128.58$ & $199.9 \pm 71.77(P=0.01)^{*}$ & $178.9 \pm 52.88(P=0.01)^{*}$ & $214.9 \pm 90.10(P=0.03)^{*}$ \\
Average NSD height $(\mu \mathrm{m}) \pm$ SD & $151.8 \pm 110.20$ & $57.5 \pm 65.52(P=0.01)^{*}$ & $24 \pm 39.55(P=0.01)^{*}$ & $63.6 \pm 91.88(P=0.01)^{*}$ \\
Average subfoveal CT $(\mu \mathrm{m}) \pm$ SD & $237 \pm 62.09$ & $220.7 \pm 46.09(P=0.14)$ & $204.9 \pm 43.12(P=0.07)$ & $205.7 \pm 36.11(P=0.03)^{*}$ \\
\hline
\end{tabular}

Notes: *Statistically significant when compared to baseline. All $P$-values are with respect to baseline.

Abbreviations: BCVA, best-corrected visual acuity; CMT, central macular thickness; CT, choroidal thickness; ETDRS, Early Treatment Diabetic Retinopathy Study; logMAR, logarithm of the minimum angle of resolution; NSD, neurosensory detachment; SD, standard deviation. 


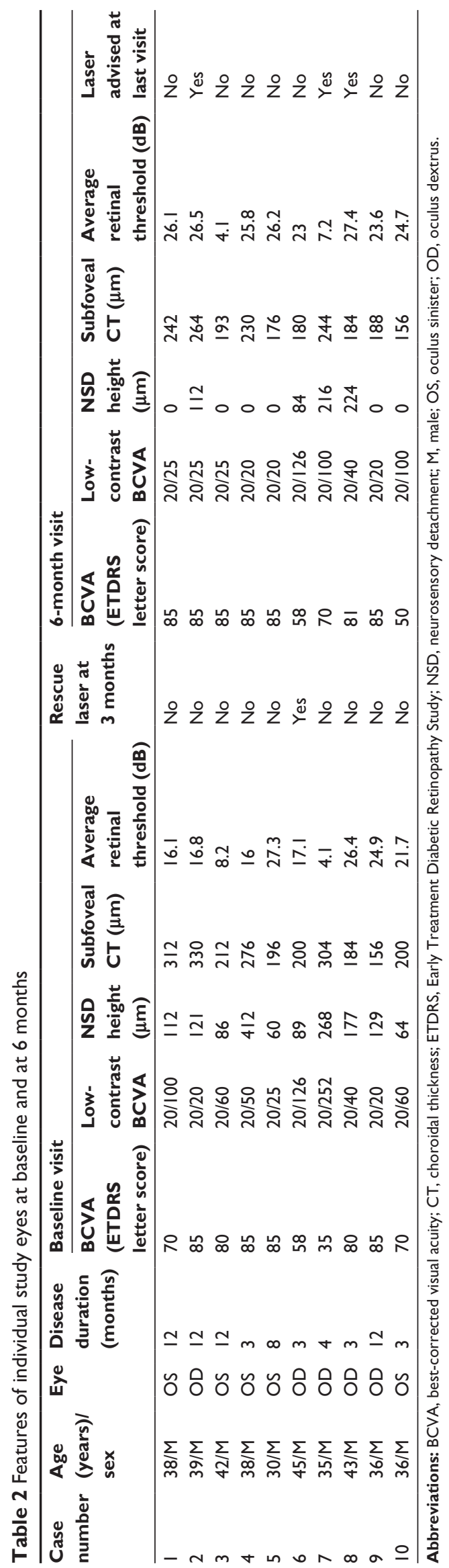

decrease in the height of NSD and was therefore given rescue laser. This eye however did not show any decrease in the height of NSD even at 6 months from baseline.

At 6 months, six (60\%) eyes showed complete resolution of SRF. However, one of these six eyes had developed an extrafoveal pocket of SRF with an extrafoveal leak on FFA, which was treated with microsecond laser at that site. Of the remaining four eyes, two eyes had shown recurrence of SRF after complete resolution at previous visit, and two eyes had continued to have persistent SRF from baseline visit.

Overall, the NSD height was significantly decreased from $151.8 \pm 110.20 \mu \mathrm{m}$ at baseline to $57.5 \pm 65.52 \mu \mathrm{m}$ $(P=0.01)$ at 1 month, $24 \pm 39.55 \mu \mathrm{m}(P=0.01)$ at 3 months, and $63.6 \pm 91.88 \mu \mathrm{m}(P=0.01)$ at 6 months. Similarly the CMT was significantly reduced from $298 \pm 128.58 \mu \mathrm{m}$ at baseline to $199.9 \pm 71.77 \mu \mathrm{m}(P=0.01)$ at 1 month, $178.9 \pm 52.88 \mu \mathrm{m}$ $(P=0.01)$ at 3 months, and $214.9 \pm 90.10 \mu \mathrm{m}(P=0.03)$ at 6 months.

There was a statistically significant thinning of choroid from $237 \pm 62.09 \mu \mathrm{m}$ at baseline to $205.7 \pm 36.11 \mu \mathrm{m}(P=0.03)$ after 6 months.

Mean inner segment/outer segment (IS/OS) junction and external limiting membrane loss at baseline were $41 \% \pm 0.46 \%$ and $24 \% \pm 0.46 \%$, respectively. At 6 -month follow-up, this significantly improved to $24 \% \pm 0.40 \%$ $(P<0.01)$ and $8 \% \pm 0.22 \%(P<0.01)$ for IS/OS junction loss and external limiting membrane, respectively.

\section{Safety}

No laser spots were visualized on any postlaser visits by biomicroscopy, SD-OCT, or on fundus autofluorescence. None of the patients had any procedure-related complications.

\section{Discussion}

Subthreshold diode laser micropulse photocoagulation has been previously used in the management of CSC, ${ }^{19-21}$ but there is no study reported on the use of $577 \mathrm{~nm}$ microsecond laser in treating subfoveal leaks in CSC. The yellow $(577 \mathrm{~nm})$ wavelength has the advantage of not being within the absorption spectrum of retinal xanthophylls, because of which, it has a potential to be used to treat subfoveal or juxtafoveal lesions. ${ }^{22}$ Moreover, this wavelength is absorbed by both melanin and oxyhemoglobin, as a consequence of which, it is less scattered compared to conventional $532 \mathrm{~nm}$ laser, and thus can deliver a more concentrated energy to a smaller volume of tissue at a lower power setting and a shorter duty cycle. 

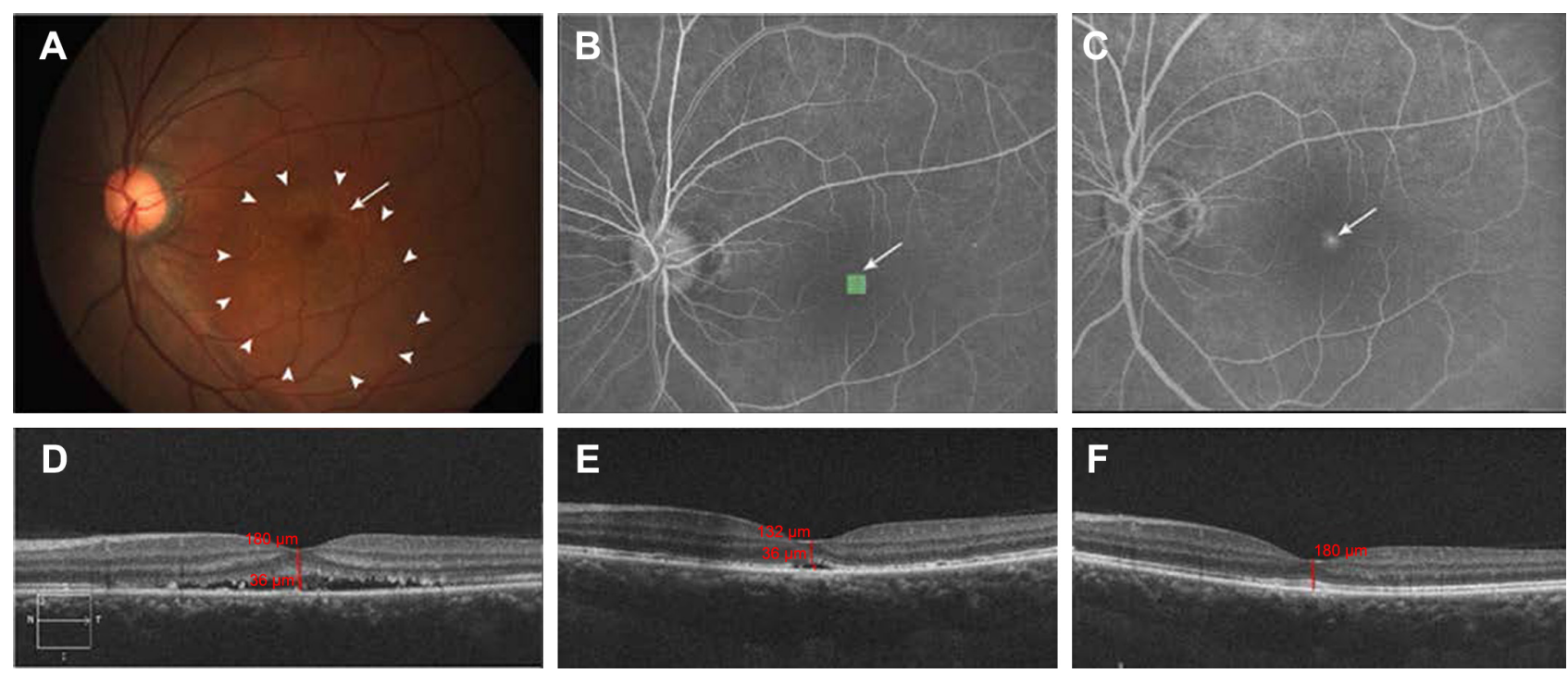

Figure I A 42-year-old male presented with blurring of vision for the past 6 months in his left eye and was diagnosed with central serous chorioretinopathy. Notes: His BCVA and LCVA were 80 letters on ETDRS chart and 20/60, respectively. (A) Color fundus photograph of the left eye showing presence of shallow subretinal fluid (arrowheads) and subretinal precipitates (arrow). Fluorescein angiography (B and C) shows the presence of subfoveal leak (arrow in C). Baseline SD-OCT shows presence of neurosensory detachment with hyper-reflective spots suggestive of chronic subretinal fluid (D). Navigated microsecond laser (5\% duty cycle) with confluent burns with $340 \mathrm{~mW}$ was performed (arrow in B). At I-month follow-up, the height of neurosensory detachment was reduced (E) and complete resolution was noted at 6 months (F). At 6-month follow-up, his BCVA and LCVA were 85 ETDRS letters and 20/25, respectively. His threshold sensitivity on microperimetry improved significantly from $16 \mathrm{~dB}$ at baseline to $25.8 \mathrm{~dB}$ at 6-month follow-up.

Abbreviations: BCVA, best-corrected visual acuity; ETDRS, Early Treatment Diabetic Retinopathy Study; LCVA, low-contrast visual acuity; SD-OCT, spectral domain optical coherence tomography.

We used microperimetry to assess the RS which is a measure of the functional outcome after treatment with microsecond laser. When compared to microperimetry, the mere assessment of BCVA has been shown to underestimate the effectiveness of photodynamic therapy in the treatment of CSC.${ }^{23}$ In our study also there was a significant improvement in the RS at 3 and 6 months from baseline, even though there was no statistically significant improvement in the BCVA and low-contrast BCVA. Previous studies have related RS on microperimetry to the status of the retinal microstructure on OCT after resolution of $\mathrm{CSC}^{24-26} \mathrm{RS}$ has been found to decrease with disruption in the RPE line and the IS/OS line, ${ }^{25}$ and found to be higher in eyes with intact IS/OS line and intact cone outer segment tips. ${ }^{27}$ Therefore, the improvement in the average retinal threshold in our study of eyes not only proves the improvement in the functional status of the retina, but also rules out structural damage to the retinal microstructure after microsecond laser.

We treated eyes that had SRF for a duration of 3 months or more. We found a rapid response in $40 \%$ of these eyes within 1 month and in $60 \%$ eyes within 6 months after treatment. It implies that microsecond laser was effective in treating these eyes, and the effect was not by chance. Two of the ten eyes, in which NSD resolved completely at 3 months, showed recurrence of SRF at 6 months and required second sitting of microsecond laser, the treatment outcome of which is yet to be evaluated. We infer that microsecond laser is effective in treating CSC with subfoveal leak but the condition may recur and require repeat laser.

Navilas, due to its ability of eye tracking and utilizing computerized laser planning on the FFA image on the same device, can perform precise targeted treatments and thus is a good alternative to the conventional manually directed laser photocoagulation in CSC, especially for subfoveal and juxtafoveal leaks. This is all the more useful in case of micropulse laser, which does not cause visible burns. For future reference, the detailed treatment report obtained from Navilas provides the exact location of the previously applied laser spots, which do not cause visible scarring as they do in the case of conventional laser.

By using the enhanced depth imaging-OCT technique, it has been reported that subfoveal $\mathrm{CT}$ is increased in eyes with CSC compared to normal eyes. ${ }^{27-29}$ Our study showed a significant progressive thinning of subfoveal choroid after 6 months, which is again indicative of the gradual resolution of the condition after microsecond laser.

Limitations of our study include a small sample size, which can be explained by the rarity of occurrence of subfoveal leak as a cause of persistent CSC. We did not have a control arm as placebo. However, resolution of persistent 
fluid with a mean of 7-month duration after microsecond laser is unlikely to occur by chance. It has been reported that eyes with CSC start developing photoreceptor atrophy as early as 4 months after the onset of symptoms. ${ }^{30}$ Further studies are required to assess if earlier intervention can cause better visual outcome. We analyzed only the B scan to evaluate the reduction in SRF. An en-face OCT imaging could have been better to understand the overall reduction in size of SRF. However, B scan is the commonly used modality in clinical practice, rather than an en-face OCT.

\section{Conclusion}

Microsecond laser is a safe and effective modality for treating cases of nonresolving CSC with subfoveal leaks. We report significant improvement in RS after microsecond laser in spite of no significant benefit in visual acuity. However, repeat micropulse laser may be required for treating cases that have recurrence. Further studies with larger sample size and longer follow-up will explore the efficacy and outcome of retreatment with microsecond laser in this distinct situation.

\section{Disclosure}

The authors report no conflicts of interest in this work.

\section{References}

1. Wang M, Munch IC, Hasler PW, Prunte C, Larsen M. Central serous chorioretinopathy. Acta Ophthalmol. 2008;86(2):126-145.

2. Gilbert CM, Owens SL, Smith PD, Fine SL. Long-term follow-up of central serous chorioretinopathy. Br J Ophthalmol. 1984;68(11): $815-820$.

3. Loo RH, Scott IU, Flynn HW Jr, et al. Factors associated with reduced visual acuity during long-term follow-up of patients with idiopathic central serous chorioretinopathy. Retina. 2002;22(1):19-24.

4. Bujarborua D. Long-term follow-up of idiopathic central serous chorioretinopathy without laser. Acta Ophthalmol Scand. 2001;79(4): 417-421.

5. Khosla PK, Rana SS, Tewari HK, Azad RU, Talwar D. Evaluation of visual function following argon laser photocoagulation in central serous retinopathy. Ophthalmic Surg Lasers. 1997;28(8):693-697.

6. Burumcek E, Mudun A, Karacorlu S, Arslan MO. Laser photocoagulation for persistent central serous retinopathy: results of long-term follow-up. Ophthalmology. 1997;104(4):616-622.

7. Tseng CC, Chen SN. Long-term efficacy of half-dose photodynamic therapy on chronic central serous chorioretinopathy. Br J Ophthalmol. 2015;99(8):1070-1077.

8. Lim JI, Glassman AR, Aiello LP, et al; Macula Society CSC Collaborative Study Group, Research and Education Committee and Website Committee. Collaborative retrospective macula society study of photodynamic therapy for chronic central serous chorioretinopathy. Ophthalmology. 2014;121(5):1073-1078.

9. Lai TY, Chan WM, Lam DS. Transient reduction in retinal function revealed by multifocal electroretinogram after photodynamic therapy. Am J Ophthalmol. 2004;137(5):826-833.

10. Colucciello M. Choroidal neovascularization complicating photodynamic therapy for central serous retinopathy. Retina. 2006;26(2):239-242.
11. Fujita K, Imamura Y, Shinoda K, et al. One-year outcomes with halfdose verteporfin photodynamic therapy for chronic central serous chorioretinopathy. Ophthalmology. 2015;122(3):555-561.

12. Luttrull JK, Sramek C, Palanker D, Spink CJ, Musch DC. Long-term safety, high-resolution imaging, and tissue temperature modeling of subvisible diode micropulse photocoagulation for retinovascular macular edema. Retina. 2012;32(2):375-386.

13. Vujosevic S, Martini F, Convento E, et al. Subthreshold laser therapy for diabetic macular edema: metabolic and safety issues. Curr Med Chem. 2013;20(26):3267-3271.

14. Glaser BM, Campochiaro PA, Davis JL, Jerdan JA. Retinal pigment epithelial cells release inhibitors of neovascularization. Ophthalmology. 1987;94(7):780-784.

15. Barnstable CJ, Tombran-Tink J. Neuroprotective and antiangiogenic actions of PEDF in the eye: molecular targets and therapeutic potential. Prog Ret Eye Res. 2004;23(5):561-577.

16. Daruich A, Matet A, Dirani A, et al. Serous chorioretinopathy: recent findings and new physiopathology hypothesis. Prog Ret Eye Res. 2015;48: $82-118$.

17. Grading diabetic retinopathy from stereoscopic color fundus photographs - an extension of the modified Airlie House classification. ETDRS report number 10. Early Treatment Diabetic Retinopathy Study Research Group. Ophthalmology. 1991;98(5 Suppl):786-806.

18. Spaide RF, Koizumi H, Pozzoni MC. Enhanced depth imaging spectral-domain optical coherence tomography. Am J Ophthalmol. 2008;146(4):496-500.

19. Ricci F, Missiroli F, Regine F, Grossi M, Dorin G. Indocyanine green enhanced subthreshold diode-laser micropulse photocoagulation treatment of chronic central serous chorioretinopathy. Graefes Arch Clin Exp Ophthalmol. 2009;247(5):597-607.

20. Lanzetta P, Furlan F, Morgante L, Veritti D, Bandello F. Nonvisible subthreshold micropulse diode laser $(810 \mathrm{~nm})$ treatment of central serous chorioretinopathy. A pilot study. Eur J Ophthalmol. 2008;18(6): 934-940.

21. Chen SN, Hwang JF, Tseng LF, Lin CJ. Subthreshold diode micropulse photocoagulation for the treatment of chronic central serous chorioretinopathy with juxtafoveal leakage. Ophthalmology. 2008;115(12): 2229-2234.

22. Joondeph BC, Joondeph HC, Blair NP. Retinal macroaneurysms treated with the yellow dye laser. Retina. 1989;9(3):187-192.

23. Ehrlich R, Mawer NP, Mody CH, Brand CS, Squirrell D. Visual function following photodynamic therapy for central serous chorioretinopathy: a comparison of automated macular microperimetry versus best-corrected visual acuity. Clin Experiment Ophthalmol. 2012;40(1):e32-e39.

24. Vasconcelos H, Marques I, Santos AR, et al. Long-term chorioretinal changes after photodynamic therapy for chronic central serous chorioretinopathy. Graefes Arch Clin Exp Ophthalmol. 2013;251(7): 1697-1705.

25. Ojima Y, Tsujikawa A, Hangai M, et al. Retinal sensitivity measured with the micro perimeter 1 after resolution of central serous chorioretinopathy. Am J Ophthalmol. 2008;146(1):77-84.

26. Fujita K, Shinoda K, Imamura Y, et al. Correlation of integrity of cone outer segment tips line with retinal sensitivity after half-dose photodynamic therapy for chronic central serous chorioretinopathy. Am J Ophthalmol. 2012;154(3):579-585.

27. Maruko I, Iida T, Sugano Y, Ojima A, Ogasawara M, Spaide RF. Subfoveal choroidal thickness after treatment of central serous chorioretinopathy. Ophthalmology. 2010;117(9):1792-1799.

28. Imamura Y, Fujiwara T, Margolis R, Spaide RF. Enhanced depth imaging optical coherence tomography of the choroid in central serous chorioretinopathy. Retina. 2009;29(10):1469-1473.

29. Brandl C, Helbig H, Gamulescu MA. Choroidal thickness measurements during central serous chorioretinopathy treatment. Int Ophthalmol.2014; 34(1):7-13.

30. Gass JD. Pathogenesis of disciform detachment of the neuroepithelium. Am J Ophthalmol. 1967;63(3 Suppl):1-139. 


\section{Publish your work in this journal}

Clinical Ophthalmology is an international, peer-reviewed journal covering all subspecialties within ophthalmology. Key topics include: Optometry; Visual science; Pharmacology and drug therapy in eye diseases; Basic Sciences; Primary and Secondary eye care; Patien Safety and Quality of Care Improvements. This journal is indexed on

Submit your manuscript here: http://www.dovepress.com/clinical-ophthalmology-journal
Dovepress

PubMed Central and CAS, and is the official journal of The Society of Clinical Ophthalmology (SCO). The manuscript management system is completely online and includes a very quick and fair peer-review system, which is all easy to use. Visit http://www.dovepress.com/ testimonials.php to read real quotes from published authors. 